\title{
Perú 2020: ¿El Quiebre de la CONTINUIDAD?
}

Peru 2020: the Interruption of Political Continuity?

\section{EDUARDO DARGENT BOCANEGRA}

Pontificia Universidad Católica, Perú

\section{STÉPHANIE ROUSSEAU}

Pontificia Universidad Católica, Perú

\begin{abstract}
RESUMEN
El Perú post-transición democrática del año 2000 se ha descrito en distintos trabajos como un caso donde se observaban algunas significativas continuidades: estabilidad del modelo económico adoptado en los noventa, un poder Ejecutivo que controla la política pública, un Congreso relativamente débil frente al Ejecutivo, ausencia de protestas articuladas, entre otras. En el periodo de gobierno (20162020) se ha dado un cambio significativo en la relación entre el Poder Ejecutivo y el Congreso que cambia este diagnóstico y podría afectar otras dimensiones de la continuidad. En general, el Poder Ejecutivo y sus tecnocracias han perdido fuerza frente a un Congreso que ha alcanzado mayor protagonismo, tanto en su control de presupuesto como por hacer más común el uso de procesos parlamentarios, como la censura ministerial y la vacancia presidencial. Estas tendencias se profundizaron en el nuevo Congreso elegido en 2020 tras la disolución del anterior. Dicho Congreso vacó al expresidente Vizcarra, fue más propenso al gasto populista y más crítico con el modelo económico, todo ello en medio de una pandemia que golpeó a Perú con dureza.
\end{abstract}

Palabras clave: Perú, Constitución 1993, Poder Ejecutivo, Poder Legislativo, COVID-19.

\begin{abstract}
After Peru's democratic transition in 2000, analysts have described the country as a case of significant political continuity. Among other aspects, the economic model adopted in the 90's was left untouched, public policy was controlled by the Executive, a relatively weak Congress meant the predominance of the Executive, and there was no broad-scale social protests at the national level. During the 2016-2020 period, a significant change occurred in the Executive-Legislative power dynamics. This transformation could affect other dimensions of the 20-year long political continuity. Generally speaking, the Executive and its technocrats have lost strength in relation to Congress' greater protagonism. The latter has managed to obtain greater control over budget, and has started to use regularly some parliamentary prerogatives such as the vote of no confidence and presidential impeachment. These tendencies continued to consolidate with the new Congress elected in 2020 after the dissolution of the preceding one by President Vizcarra. The latter was in turn impeached by the new Congress. The year 2020 was marked by a greater propensity by Congress to promote populist measures and criticize the existing economic model, in the midst of a pandemic that strongly hit Peru and affected the country harshly.
\end{abstract}

Keywords: Peru, 1993 Constitution, Executive Power, Legislative Power, COVID-19.

DOI: $10.4067 /$ S0718-090X2021005000112 


\section{INTRODUCCIÓN}

El Perú post-transición democrática del año 2000 ha sido descrito en distintos trabajos académicos como un caso donde se observaban algunas significativas continuidades que contrastan con procesos de cambio en América Latina (Tanaka y Vera 2008; Dargent y Muñoz 2012). Un país donde el modelo económico instaurado en los noventa no fue modificado sustantivamente por distintas razones, como el impacto territorial diferenciado de la economía o el considerable poder de actores empresariales (financieros, exportadores, mineros) que ganaron con las reformas de mercado (Durand 2006; Awapara 2018). En el que la debilidad partidaria, atribuida en los noventa al autoritarismo de Alberto Fujimori (1990-2000), se mantuvo elección tras elección en democracia (Levitsky y Cameron 2003; Zavaleta 2014). Un país donde se observan numerosos conflictos locales, con frecuencia vinculados a temas socioambientales, pero que no escalan a nivel nacional ni se articulan bajo movimientos más comprensivos contra el modelo económico. Un caso donde el Ejecutivo y sectores tecnocráticos del Estado mantenían cierto control sobre el Congreso y la política pública en general (Meléndez y León 2010; Dargent 2015; Vergara y Encinas 2016) a pesar de la debilidad de los partidos de gobierno y la conflictividad local antes señalada. Si tuvieramos que elegir entre cambio y continuidad, el Perú post-transición era más un caso de continuidad, algo sorprendente por la debilidad de los actores políticos que harían pensar que observaríamos más inestabilidad y cambio.

En varios de estos trabajos, sin embargo, es precisamente la debilidad de los actores políticos la causa que explica la continuidad de algunos de los fenómenos mencionados. El Ejecutivo lograba mantener cierta estabilidad y continuidad por su mayor fortaleza relativa frente a los partidos de oposición en el Congreso. Asimismo, actores empresariales favorecidos por el modelo económico y fortalecidos en estos años, defienden la continuidad del modelo frente a partidos críticos del mismo con menos poder e incapaces de articular intereses a través del territorio. El Ejecutivo ha defendido dicha continuidad económica, sea por convicción o por estrategia, al no querer enfrentar la crítica de estos actores privados con mayor poder. El caso más claro es el de Ollanta Humala, que gana la elección en 2011 con una propuesta de cambio pero que rápidamente acepta los términos del establishment económico para ganar estabilidad.

En el periodo de gobierno (2016-2020) una de estas "continuidades" se ha quebrado, dejando abierta la pregunta de cómo se afectarán las otras en el futuro. El Poder Ejecutivo ha perdido fuerza frente a un Congreso que ha alcanzado mayor protagonismo. Se ha generalizado el uso de procesos parlamentarios de control horizontal antes poco utilizados, como la censura ministerial y la vacancia presidencial por el causal de incapacidad moral permanente. Y esa pérdida de poder del Ejecutivo ha significado la pérdida de control económico y político de élites tecnocráticas sobre los políticos tanto en el Congreso como en las regiones. En particular, el Ministerio de Economía y la Presidencia del 
Consejo de Ministros ya no tienen la influencia de años anteriores y su posibilidad de veto se ha reducido.

Este debilitamiento del Ejecutivo, como veremos en la cuarta sección, se explica en parte por el agrio conflicto entre el gobierno y la bancada de Fuerza Popular producido tras la elección de Pedro Pablo Kuczynski (2016-2018), pero también por la extrema debilidad del partido de gobierno en el Congreso. Kuczynski ganó en la segunda vuelta la presidencia a Keiko Fujimori por apenas $0.24 \%$ de los votos (50.12\% vs $49.88 \%$ ). En primera vuelta, sin embargo, Fujimori fue clara ganadora con $39.9 \%$ de los votos y contó con una amplia mayoría en el Congreso (73 escaños, 56\% del total) mientras que Kuczynski apenas obtuvo $21 \%$ en la elección presidencial y una pequeña bancada de 18 congresistas (14\%) (Dargent y Muñoz 2016). Fujimori demoró en aceptar los resultados de la elección y su bancada atacó duramente al Ejecutivo con recursos como la interpelación y censura a ministros, así como la amenaza de vacancia. El gobierno no tenía la capacidad, ni siquiera formando alianzas, de reducir esta amenaza a su estabilidad. El conflicto iniciado por esta mayoría en el Congreso contra el Ejecutivo, en un país atravesado por escándalos de corrupción vinculados al caso Lava Jato y otros, terminó con la Presidencia de Kuczynski, quien tuvo que renunciar en marzo del 2018 antes de que se votase una segura vacancia presidencial (Ponce de León y García 2019).

La llegada del vicepresidente Martín Vizcarra al poder dio lugar a una nueva dinámica de enfrentamiento de la Presidencia contra el Congreso, pero esta vez con un Presidente que gozó de mayor apoyo de la ciudadanía por adoptar una agenda anticorrupción. El resultado tras una serie de escaramuzas y amenazas mutuas fue el cierre del Congreso en noviembre del 2019, utilizando la figura constitucional de la disolución en forma controversial (aunque luego ratificada por el Tribunal Constitucional). En el camino cambiaron la forma en que se habían desarrollado las relaciones Ejecutivo-Legislativo hasta entonces, como también creció el peso del Congreso en la política y la política pública. El sistema político en el 2019 no era el mismo del 2016 aunque las reglas constitucionales siguieran siendo las mismas.

Este artículo se centra en lo sucedido el 2020, otro año desbocado de la política peruana donde esta debilidad del poder del Ejecutivo frente al legislativo se profundizó, brindando una mirada a sus antecedentes y sus posibles consecuencias. El Congreso mantuvo su poder y el Ejecutivo incrementó su precariedad, a pesar de ya no haber una mayoría hegemónica en control del legislativo. Con un Congreso fragmentado, vimos similares dinámicas de conflicto e incluso un mayor contenido populista y de crítica a la forma en que se ha manejado la economía al aprobar leyes que se consideraron invasivas de competencias del Ejecutivo y otras que afectaban el déficit fiscal. Este Congreso además pudo actuar con mayor libertad al carecer el Ejecutivo de una bancada propia (Vizcarra no apoyó a pesar de su popularidad a ninguna agrupación en campaña) y también de armas institucionales de disuasión, pues la Constitución no permite la disolución del Congreso en su último año de ejercicio. Detallamos los diver- 
sos conflictos entre el gobierno y el Congreso que llevaron a la vacancia del propio Martín Vizcarra en noviembre, la caída del breve gobierno de Manuel Merino, presidente del nuevo Congreso, tras masivas protestas nacionales en su contra, y el nombramiento de Francisco Sagasti, nuestro cuarto presidente en este periodo de gobierno. Finalmente, también discutimos cómo las movilizaciones políticas de protesta por la vacancia de Vizcarra fueron masivas y a través del territorio, cuestionando otro sentido común de la continuidad: que el Perú es un país con baja articulación en la protesta. Además, para cerrar el año, en diciembre se dieron movilizaciones en varios puntos del país contra un régimen legal de excepción que promueve la agroexportación. Tras politizarse el tema, y una serie de bloqueos de carretera en valles costeros, se reformó el régimen especial, mostrando que aspectos básicos del modelo económico de los noventa se han puesto en discusión. Es muy pronto si en ese frente estamos ante un cambio sustantivo o solamente a una reacción por la crispación política de un año muy difícil en términos políticos.

El artículo tiene la siguiente estructura. Tras una revisión del contexto socioeconómico del año 2020 que nos da un marco sobre el severo impacto del Covid-19 en el país, se presenta en la tercera sección los antecedentes que dieron lugar a la elección de un nuevo Congreso en enero, el conflicto entre Ejecutivo y Legislativo, la vacancia de Vizcarra y las protestas de noviembre. En la cuarta sección exploramos las causas que llevaron al debilitamiento del Poder Ejecutivo en el quinquenio y en este año en particular. En la conclusión discutimos los escenarios que se abren para el próximo periodo presidencial. Si bien es muy pronto para saber si este quiebre de la continuidad en el tipo de relación Ejecutivo-Legislativo traerá otros cambios en las dinámicas políticas en el Perú, un Ejecutivo que continúe en esta posición de debilidad hace pensar en que otras dimensiones seguirán viéndose afectadas.

\section{CONTEXTO SOCIO-ECONÓMICO DEL 2020}

El año 2020 empezó con una situación socioeconómica relativamente estable, habiendo terminado el 2019 con un crecimiento del PIB de 2.2\%. Sin embargo, las consecuencias económicas de la pandemia del COVID-19 se hicieron sentir desde las primeras semanas luego de la declaratoria del estado de emergencia sanitaria el 11 de marzo $2020^{1}$. De uno de los países latinoamericanos con la mejor performance económica durante varios años, el Perú pasa a ser el que mayor caída sufre luego de Venezuela, según un estudio preliminar de la CEPAL. Al finalizar el primer trimestre del 2020 se registra una contracción muy importante, con un PIB de $-3.4 \%$ (ver gráfico 1). Esta recesión se profundiza a lo largo del año para llegar a -9.4\% en el tercer trimestre (INEI 2021). Según la CEPAL, Perú sufrió una recesión de $12.9 \%$ del PIB en total el 2020 (CEPAL 2021: 124).

DECRETO SUPREMO Nº08-2020-SA. Decreto Supremo que declara en Emergencia Sanitaria a nivel nacional por el plazo de noventa (90) días calendario y dicta medidas de prevención y control del COVID-19. 
Gráfico 1. PBI Real 2010-2020

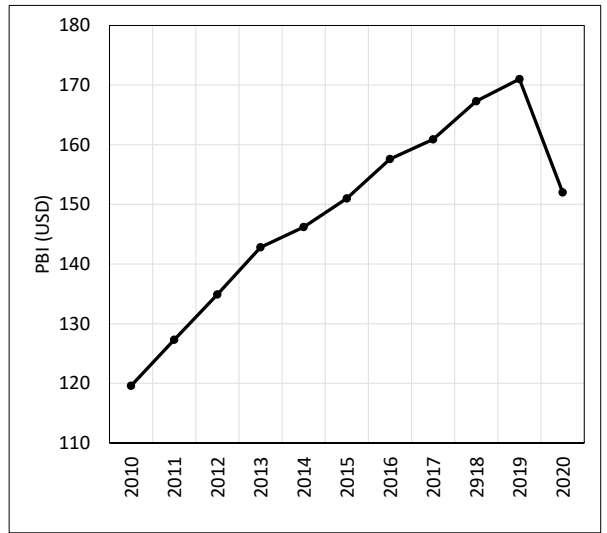

Fuente: Elaboración propia en base a datos del Banco Central de Reserva del Perú (BCRP).

La recesión significó una importante pérdida de empleo en el sector formal, debido a la inmovilización social obligatoria, la caída de las inversiones extranjeras, y un consumo en franco declive. Además, la inmovilización social obligatoria afectó también severamente la actividad económica informal, en la cual se encuentra aproximadamente el 70\% de la población activa. La tasa de participación económica global pasó de $72.5 \%$ en 2019 a 59.6\% de la población en edad de trabajar en 2020. Al mismo tiempo, la brecha de género en la participación económica se incrementó de 15\% a 20\% (CEPAL 2021: 140). En los dos últimos meses de 2020 y en enero 2021, la población ocupada de Lima Metropolitana disminuyó en $11.9 \%$, en comparación con el mismo periodo un año antes (INEI 2021) (ver gráfico 2). Se estima que el trabajo informal aumentó en aproximadamente $10 \%$ a raíz de la pandemia (Lavado y Liendo 2020).

Gráfico 2. Desempleo de 2010 a 2020

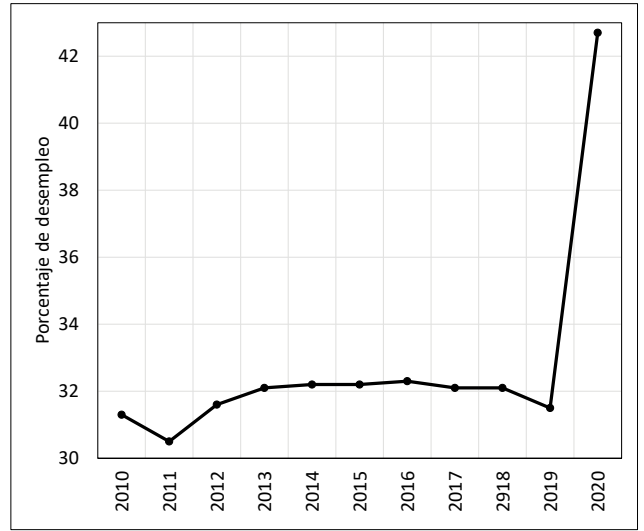

Fuente: Elaboración propia en base a datos de la ENAHO. 
Como consecuencia de la recesión, la sociedad peruana enfrentó un retroceso brutal en cuanto a los niveles de pobreza y pobreza extrema. Los logros de los últimos diez años en la reducción de la pobreza se han evaporado. Lavado y Liendo (2020) estimaban un regreso al nivel de pobreza monetaria del 2010 (aproximadamente 30\% de la población), lo cual fue prácticamente confirmado por las cifras del INEI: 30.1\% de la población se encontraba en situación de pobreza para el 2020, muy cerca del 30.8\% de 2010. Por su parte, la CEPAL (2020: 9) estimaba que la proporción de la población en situación de pobreza extrema aumentaría 4 puntos porcentuales en relación a la cifra de 2019 (3.7\% de la población) (ver gráfico 3).

El desempleo creció de manera abrupta y dio lugar a una significativa ola de migración interna. A pocas semanas de iniciada la inmovilización social obligatoria, más de 167,000 peruanos y peruanas se vieron obligados a salir de la ciudad, adonde habían migrado meses o años atrás (Berganza 2020). Al buscar volver a sus pueblos de origen, pretendían escapar a su vida difícil de migrante que ya no les permitía asumir los gastos corrientes de la vida urbana. Sin recursos propios, hambrientos y sin ayuda económica, estos sectores empezaron a acampar a la espera de encontrar un medio de transporte; miles se desplazaron incluso a pie, generando que se los llamara "los caminantes". Esta migración generó un contexto de alto riesgo de contagio para las localidades receptoras, además de los propios migrantes que muchas veces se desplazaban en grupos grandes.

Gráfico 3. Pobreza y pobreza extrema 2010-2020

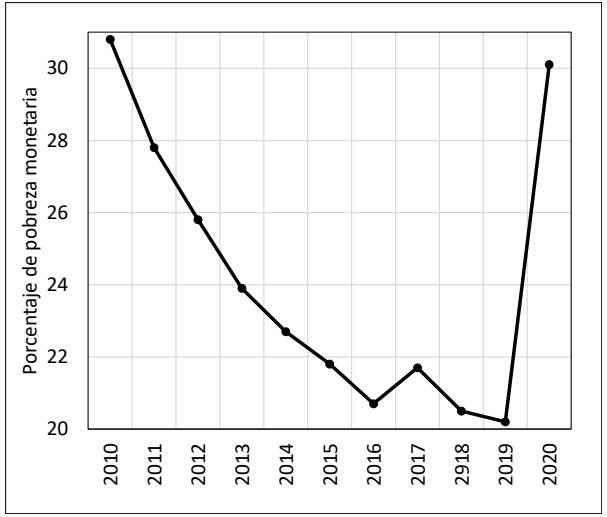

Fuente: Elaboración propia en base a datos del INEI.

En la capital y en las otras grandes ciudades del país, las condiciones de vida precarias de muchos sectores de la población fueron causantes de mayor riesgo de contagio, debido a la necesidad de salir de su domicilio para comprar alimentos diariamente en mercados sobrepoblados. Para muchos, la obligación de salir a trabajar para sobrevivir se volvió un problema serio, dado el riesgo de 
contagio y la amenaza de ser detenido por las fuerzas del orden encargadas de asegurar el cumplimiento de las medidas de inmovilización social. Durante los meses de reactivación económica en particular, la informalidad del transporte urbano también resultó ser un vector importante de transmisión del Covid-19. Incluso en los vehículos más formales del sistema de bus y tren metropolitano, la congestión y colas interminables fueron materia de preocupación para la población y las autoridades. El control de aforo resultó ser otro problema, esta vez para las empresas de transporte que argumentaron no poder mantener sus actividades con una caída tan drástica de ingresos.

No podemos cerrar este balance de la coyuntura socioeconómica del 2020 sin referirnos a la situación sanitaria como tal. La llegada de la pandemia al Perú ha sido catastrófica y ha producido rápidamente un colapso del sistema de salud. Tal como se podía prever en un país cuyo gasto público en salud ha sido sistemáticamente por debajo del promedio latinoamericano ${ }^{2}$, el sistema de atención de emergencia y cuidados intensivos no estaba preparado ni en equipamiento de protección del personal de salud, ni en equipos para atender la emergencia, como ventiladores, plantas de oxígeno, o número de camas en unidades de cuidados intensivos. Al principio de la pandemia, Perú contaba con 0.9 camas UCI por 100,000 habitantes, lejos del mínimo de 6 considerado por los especialistas en medicina intensiva. A mediados del año 2020, se logró alcanzar unas 4.9 camas UCI por 100,000 habitantes, todavía por debajo del mínimo de 6 camas. En comparación, al mismo momento Colombia disponía de 6.8 camas UCI por 100,000 habitantes, y Chile superaba ampliamente el estándar mínimo con 12 camas (Salud con Lupa 2017).

La situación en cuanto a la disponibilidad de oxígeno medicinal también mostró las fallas del sistema de salud. La producción de oxígeno medicinal estaba concentrada en solamente dos empresas privadas, lo cual significaba una baja capacidad de abasto, además de representar un casi monopolio y por lo tanto un precio muy alto del oxígeno. ${ }^{3}$ Adicionalmente, las normas sanitarias habían establecido que solo el oxígeno con el mayor nivel de pureza podía ser administrado en los hospitales públicos, cuando niveles menores de pureza son adecuados según estándares internacionales. Esta norma parecía ser parte de las razones por las cuales se mantenía el monopolio. La escasez de oxígeno medicinal generó un mercado informal de venta de balones al por menor, cuya calidad no podía ser garantizada (Defensoría del Pueblo 2020).

Por otro lado, sin capacidad de rastreo de contactos de COVID-19, la relativa eficiencia del sistema sanitario en aplicar pruebas rápidas de descarte ha sido

Por ejemplo, en 2017, el gasto público en salud en porcentaje del PIB era 3.2\% en Perú, mientras el promedio latinoamericano era 4.3\%. Ver Podestá (2020:33).

En 2010, mediante resolución de la Sala Especializada en Defensa de la Competencia del Instituto Nacional de Defensa de la Competencia y de la Protección de la Propiedad Intelectual, las dos empresas habían sido sancionadas por prácticas de reparto de mercado. Resolución N N 051-2010/CLC-INDECOPI, 13 de agosto de 2010 . 
insuficiente para frenar el contagio. Recién a fines del 2020 se implementó una planta para fabricar pruebas moleculares en el territorio peruano, gracias a los esfuerzos de un laboratorio privado en consorcio con una universidad nacional (UNMSM 2021). La comunidad científica puso el hombro para suplir por las deficiencias del sistema de salud público, a través de la producción de ventiladores, plantas de oxígeno y otros aportes cruciales (Andina 2021).

Sin la preparación previa y con un sistema de salud fragmentado y precario, la pandemia dejó como saldo de la primera ola un número altísimo de contagiados y una tasa de letalidad entre las más altas del mundo. A diciembre se contabilizaba oficialmente más de 37,000 muertes, pero un sistema estatal de registro de fallecidos (SINADEF) mostraba un exceso de fallecidos en comparación a otros años que apuntaba a cerca de 95,000 en el año (Gestión 2020) (ver gráfico 4). Para contextualizar la tragedia, la Comisión de la Verdad y Reconciliación calcula en 69,000 las personas que murieron en el conflicto armado interno que sufrió el Perú en los ochenta y parte de los noventa. Con sus casi 33 millones de habitantes, el número de muertes oficiales colocaba, según el observatorio COVID-19 de la Universidad Johns Hopkins, a Perú como el primer país de las Américas en cuanto a la tasa de letalidad, con 106,7 muertes por 100,000 habitantes al final de octubre 2020. Lamentablemente, las autoridades no lograron solucionar varios aspectos claves de la deficiente respuesta sanitaria para poder enfrentar mejor la segunda ola, la cual se desató a partir de enero del 2021 (Herrera-Añazco et al. 2021).

Al principio del 2021, se reportaba más de 12,000 médicos contagiados y 300 médicos fallecidos por COVID (Colegio Médico del Perú 2021). El Colegio de Enfermeros reportaba aproximadamente 7,600 profesionales de enfermería y 8,000 técnicos de enfermería contagiados (RPP 2021). El malestar del personal médico por las condiciones de alta precariedad en la atención hospitalaria se hizo sentir a través de huelgas y protestas espontáneas en la salida de hospitales en diferentes momentos del año 2020. Las disparidades entre regiones en cuanto a los recursos en personal sanitario y acceso a oxigeno medicinal fue otro problema serio. Como ejemplo, podemos citar un estudio de 2015 que establecía que existía un promedio nacional de 4.61 médicos por 10,000 asegurados del sistema de salud público, pero que la variación inter-regional iba del mejor ratio, en la región de Moquegua, con 12.47 médicos por cada 10,000 asegurados, al peor ratio en Loreto con 2.48 (Oyola 2021).

En resumen, la economía peruana tuvo un enorme impacto con el COVID, bastante más alto que la mayoría de América Latina, y los sistemas de salud estuvieron muy lejos de las capacidades necesarias para responder al fenómeno. Como veremos en las siguientes secciones, este malestar ha alimentado una ya muy baja confianza ciudadana en el Estado y el sistema político en general. 
Gráfico 4. Muertes 2020 comparado con 2019

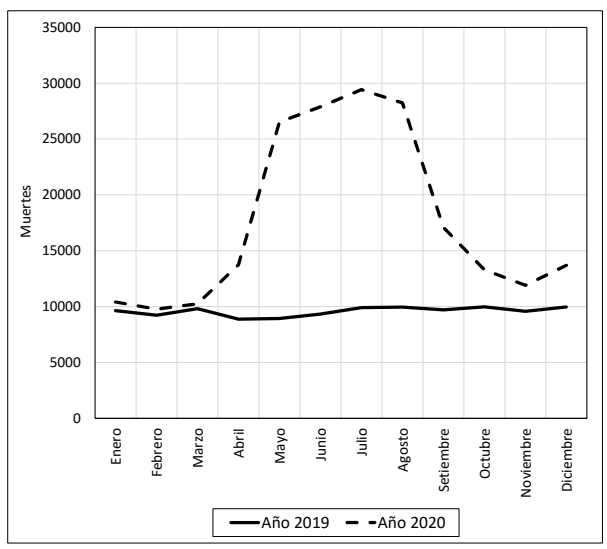

Fuente: Elaboración propia en base a datos del SINADEF.

\section{COYUNTURA POLÍTICA EN EL AÑO DE LA PANDEMIA}

A nivel político-institucional, el país ingresó al año de la pandemia en una situación mucho más frágil que en el plano socio-económico. En los años inmediatamente anteriores, una situación de enfrentamiento constante entre el Congreso y el Ejecutivo se había instalado y complejizado aún más con lo explosivo que fue el caso Lava Jato para numerosos actores partidarios, autoridades del poder judicial y expresidentes, entre otros (Paredes y Encinas 2020). La segunda mitad del año 2019 fue marcada por la voluntad del presidente Vizcarra de avanzar en la adopción de una serie de reformas políticas que su gobierno había propuesto y que habían sido sometidas a la aprobación de la población al final del 2018 a través de un referéndum. Debido al alto nivel de conflicto entre el Congreso y el Ejecutivo, a la ausencia de una bancada oficialista en el Congreso y a la mayoría fujimorista que lo controlaba, la discusión y aprobación de estas reformas se demoró y se complicó (Paredes y Encinas 2020).

La voluntad por parte del Congreso de acelerar la elección de miembros del Tribunal Constitucional fue un asunto que acentuó su enfrentamiento con el Ejecutivo, lo cual desemboco en una crisis institucional sin precedentes desde el 2000. Correspondía al Congreso renovar a seis de siete magistrados que conforman el Tribunal. El temor del Ejecutivo era que con esta nueva conformación, y especialmente dado el peso que tendría el Fujimorismo en la elección, el Tribunal pudiese convertirse en una instancia que avale los actos del Congreso, entre ellos, medidas contrarias al Ejecutivo. Eventualmente, el Tribunal podía limitar la capacidad del presidente para disolver el Congreso, el contrapeso institucional que le permitía a Vizcarra hasta entonces mantener al Congreso a raya.

El tema del Tribunal Constitucional se complica cuando el Congreso inicia un proceso acelerado de selección, aprobando una lista de candidatos preli- 
minar que respondía a los intereses de los partidos en el Congreso. Tras una accidentada sesión, el Ejecutivo plantea una cuestión de confianza contra la decisión de proceder con la elección de magistrados. El Congreso vota a favor de la cuestión de confianza, dándole la razón al Ejecutivo, pero procede con la elección de magistrados al considerar que no afectaba al proceso en curso. El Ejecutivo considera que al hacerlo el Congreso ha rechazado tácitamente la confianza solicitada y procede a disolver el Congreso en aplicación del artículo 134 de la Constitución, al ser éste el segundo gabinete al que el Congreso negaba su confianza en el periodo (el primero fue durante el gobierno de Kuczynski). Ese mismo día se vota la suspensión en el cargo del mismo presidente por parte del Congreso. Este último juramentó a la vicepresidenta en función, Mercedes Araoz.

La opinión pública se decantó rápidamente en favor del presidente en horas de incertidumbre sobre la presencia de dos presidentes. Además, Vizcarra consiguió el apoyo del Comando Conjunto de las Fuerzas Armadas, el Ejército, la Marina y la Fuerza Aérea, lo cual le permitió imponerse frente a la pretensión del Congreso de suspenderle y nos recordó el peso que todavía tienen los militares en la política peruana. Con el apoyo masivo de la población, Vizcarra convocó a elecciones legislativas extraordinarias (Paredes y Encinas 2020). Y pocas horas después Araoz renunció a su cargo. Meses después el Tribunal Constitucional declararía por cuatro votos contra tres que la disolución fue adecuada al considerar que el Congreso no podía votar por la confianza y luego actuar contra lo decidido. Por el lado opuesto, se argumentó que la figura de la confianza debe ser mucho más precisa y concisa, para evitar la posibilidad de invocar "negaciones tácitas".

De esta manera, el año 2020 empieza con elecciones congresales extraordinarias, un proceso inusual. Aunque ya estaba vigente la reforma Constitucional promovida por Vizcarra que impide la reelección de congresistas, el Jurado Nacional de Elecciones autorizó la participación de los representantes elegidos al Congreso disuelto, considerando el carácter extraordinario de estas elecciones y que estaríamos dentro del mismo periodo de gobierno (desde enero del 2019 se ha prohibido la reelección parlamentaria en el Perú). De los 130 congresistas "disueltos", 19 intentaron la reelección, y solo 2 consiguieron ser reelegidos: Alberto de Belaunde y Gino Costa, ambos ex integrantes del Grupo Parlamentario Bancada Liberal, que se destacaron por su oposición a la mayoría fujimorista. La elección, el 26 de enero, llevó a la conformación de un Congreso muy fragmentado, con nueve agrupaciones políticas representadas, en comparación con las seis que habían conseguido elegir representantes al Congreso en 2016. En 2020, ningún partido logró conseguir siquiera un tercio de los escaños. El partido Acción Popular fue el más votado, consiguiendo 25 de los 130 escaños. Cinco de los nueve partidos representados no habían ingresado en el Congreso anterior (ver Tabla 1). Sin embargo, para la casi totalidad de estos, no se debe a que no habían conseguido suficientes votos en 2016, sino simplemente porque no habían postulado. Resultó sorprendente el ingreso de 15 representantes del 
partido Frente Popular Agrícola Del Perú - FREPAP y de 13 del partido Unión Por el Perú (UPP), dada su retórica anti-sistema y su arraigo más popular en comparación con las demás fuerzas políticas. El fujimorista Fuerza Popular, por su parte, pasó de 73 a 15 representantes elegidos, una fuerte sanción a su desempeño como mayoría.

Tabla 1. Composición del Congreso 2020

\begin{tabular}{lc}
\hline Bancadas & Número de Congresistas \\
\hline Acción Popular (AP) & 25 \\
Alianza Para el Progreso (APP) & 22 \\
Somos Perú (SP) & 11 \\
Podemos Perú (PP) & 11 \\
Unión Por el Perú (UPP) & 13 \\
Fuerza Popular (FP) & 15 \\
Frepap & 15 \\
Partido Morado (PM) & 9 \\
\hline
\end{tabular}

Fuente: Elaboración propia con datos de la ONPE.

El nuevo Congreso se instaló el 17 de marzo 2020, coincidiendo con el inicio del aislamiento social obligatorio debido al COVID-19. ${ }^{4}$ Dada la corta duración del mandato de los Congresistas-con elecciones generales previstas para el 2021, era de prever, como sucedió, que la mayoría de quienes apostaron por ocupar un sillón del parlamento tendría poca experiencia previa. Más allá del carácter amateur que caracteriza al Congreso 2020-2021, debemos subrayar la gran prevalencia de intereses particulares, especialmente los vinculados a la educación universitaria privada. El carácter amateur de los representantes y los intereses particulares iniciaron desde muy temprano una dinámica de conflicto con el Ejecutivo, agudizada por la alta fragmentación política, la ausencia de una bancada de gobierno y con consecuencias aún más dramáticas tomando en cuenta la emergencia sanitaria y la crisis económica que desató.

En este contexto, los choques entre el Ejecutivo y el Congreso prevalecieron, llevando a la aprobación de varias leyes por insistencia del Congreso tras ser observadas por el Poder Ejecutivo. Leyes cuyo contenido claramente apuntaba a promover intereses particulares o satisfacer demandas inmediatas de la población en el marco de la pandemia de manera populista e imprudente (ver Tabla 2). Por ejemplo, uno de los temas dominantes en los debates del Congreso fue el sistema de pensiones. La oportunidad de permitir a los aportantes tanto del sistema público (ONP) como del privado (AFP) (en el Perú conviven los dos sistemas) retirar cierta proporción de sus aportes previsionales para disponer de efectivo en el contexto de la crisis económica, se mezcló con la ambición de 
elaborar una reforma integral del sistema..$^{5}$ Existe en la población y en la opinión de varios expertos una fuerte crítica al sistema de pensiones creado en los años noventa. Sin embargo, la situación crítica en la cual se encontraban varios sectores de la población afectados por el desempleo y el cese de varias actividades económicas fue motivo de decisiones imprudentes por parte del Congreso en relación al sistema previsional. El Poder Ejecutivo se opuso a la adopción de una Ley de retiro excepcional de parte de los aportes a las cuentas individuales de AFP. También se opuso a la adopción de una Ley que permitía el retiro de hasta 4300 soles por parte de los aportantes activos e inactivos de la ONP. En el caso de la ONP los aportantes no tienen cuentas individuales. Se trata de un fondo al que aportan los trabajadores formales en el sistema público y del que se paga a los actuales pensionistas. Las medidas adoptadas por el Congreso fueron criticadas por los expertos en temas previsionales, por el riesgo que representaban en cuanto a la estabilidad y sostenibilidad del sistema público de pensiones (CIUP 2020).

Tabla 2. Datos de leyes aprobadas por insistencia

\begin{tabular}{|c|c|c|}
\hline Ley & Fecha de votación & Fecha de observación \\
\hline $\begin{array}{l}\text { Ley } \mathrm{N}^{\circ} \text { 31018: "Ley que suspende el cobro de peajes } \\
\text { en la red vial nacional, departamental y local conce- } \\
\text { sionada, durante el estado de emergencia nacional } \\
\text { declarado a causa del brote del COVID-19 }\end{array}$ & 7 de mayo & 29 de abril \\
\hline $\begin{array}{l}\text { Ley N }{ }^{\circ} 31039 \text { : “Ley que regula los procesos de ascen- } \\
\text { so automático en el escalafón, el cambio ocupacio- } \\
\text { nal, cambio de línea de carrera, el nombramiento y } \\
\text { cambio a plazo indeterminado de los profesionales, } \\
\text { técnicos, auxiliares asistenciales y personal adminis- } \\
\text { trativo de la salud }\end{array}$ & 24 de agosto & 7 de julio \\
\hline $\begin{array}{l}\text { Ley N } \mathrm{N}^{\circ} \text { 31112: “Ley que establece el control previo de } \\
\text { operaciones de concentración empresarial }\end{array}$ & 30 de diciembre & 25 de noviembre \\
\hline
\end{tabular}

Fuente: Elaboración propia con datos del Congreso.

Estas y otras leyes aprobadas por insistencia del Congreso chocaron con las medidas adoptadas por el Ejecutivo para paliar la situación de vulnerabilidad económica en la cual cayó un gran sector de la población en 2020. En abril 2020, el Ejecutivo había enviado un proyecto de reforma del sistema de pensiones, el cual fue rechazado por el Congreso. El gobierno de Martin Vizcarra insistió en mantener separados esa reforma y las medidas de emergencia económica.

El gobierno desplegó una serie de medidas de emergencia sanitaria y económica que, al inicio de la pandemia, fueron evaluadas positivamente. Por ejemplo, Perú fue, luego de Venezuela, el país sudamericano que más rápidamente

El Congreso trabajó en una propuesta de reforma integral, a través de la creación, en mayo 2020, de la Comisión Especial Multipartidaria Encargada de Evaluar, Diseñar y Proponer el Proyecto para la Reforma Integral del Sistema Previsional Peruano. 
declaró un periodo de cuarentena obligatoria nacional luego del primer caso confirmado de COVID en su territorio (PNUD 2020). A la vez, adoptó reglas entre las más restrictivas de América Latina que al inicio contaron con el apoyo de la comunidad científica y el público. El gobierno decidió otorgar bonos de emergencia como subsidio temporal a las personas, a través del Bono Familiar Universal para hogares que no percibieron ingresos formales durante el periodo de emergencia, y de bonos para hogares clasificados como pobres o en extrema pobreza en el sistema de focalización de hogares. En septiembre, anunciaba que más de 2.5 millones de aportantes a la ONP recibirían el segundo Bono Familiar Universal. A la vez, el gobierno ampliaba el padrón de beneficiarios del bono para incluir a 1.2 millones de aportantes adicionales que no contaban con ingresos mensuales. Asimismo, se aprobó un bono extraordinario de 760 soles adicionales para 560 mil pensionistas.

Si bien el gobierno mostró una buena disposición a dedicar fondos públicos para otorgar protección social temporal a muchos sectores de la población que normalmente quedaban fuera del radar de la política social, se evidenciaron varios retos en la entrega de los subsidios. Primero, la llegada de los diferentes bonos fue tardía y segmentada innecesariamente en diferentes categorías de beneficiarios. Segundo, la logística de la entrega a la población sufrió de numerosos problemas: colas interminables, sistemas de información deficientes, y retos propios a la naturaleza de la infraestructura de transporte y restricción de movilidad. A la par, una población que en su gran mayoría está empleada informalmente debía romper las medidas sanitarias para trabajar.

Los recursos públicos fueron utilizados también para apoyar a las empresas y al sistema financiero, a través del programa Reactiva Perú y otros fondos de apoyo empresarial y agrícola. Básicamente, los programas consistían en garantizar los nuevos créditos otorgados a MYPEs y grandes empresas por el sistema financiero, para asegurar la integridad de las cadenas de pagos y los sueldos de los trabajadores.

Luego de gozar de alta aprobación popular durante los primeros meses de la pandemia, el presidente Vizcarra empezó cierto declive que fue eventualmente aprovechado por el Congreso para maniobrar un primer intento de vacancia en septiembre. A raíz de un escándalo vinculado a audios que supuestamente mostraban al presidente tratando de ocultar su rol en la contratación irregular de un cantante por el Ministerio de Cultura para que desarrollara cursos para los que no estaba calificado, se planteó una moción de vacancia por "incapacidad moral permanente".${ }^{6}$ Esto dio lugar a que, el 14 de septiembre, el Procurador Público Especializado en Materia Constitucional del Poder Ejecutivo, interpusiera una demanda competencial al Tribunal Constitucional contra el Congreso de la República y una medida cautelar solicitando detener el proceso

La Constitución política del Perú plantea en su art. 113 diferentes causales por los cuales el Congreso puede votar la vacancia presidencial. En el 113.2 se especifica la siguiente: "Su permanente incapacidad moral o física, declarada por el Congreso." 
político en el Congreso hasta que se resuelva la demanda. El Tribunal aceptó la demanda de competencia, pero no concedió la medida cautelar.

Vizcarra negó las acusaciones y declaró su disposición a colaborar con las investigaciones del caso, aunque mantuvo en suspenso si asistiría o no al Congreso a ejercer su defensa mientras el Tribunal Constitucional no decidiera el caso. Pocos días después de la demanda competencial, y sin esperar la decisión del Tribunal Constitucional, el Congreso procedió al proceso constitucional, recibiendo al presidente para que ejerza su defensa y votando la moción, que fue rechazada ampliamente. ${ }^{7}$ El presidente logró salvarse esa vez, pero allí no terminó el intento de ciertos grupos de Congresistas de deshacerse de su gobierno. A finales de octubre, a raíz de un nuevo escándalo que aparentemente involucraba a Vizcarra en un caso de corrupción en obras públicas durante su mandato de gobernador regional de Moquegua (20112014), el Congreso reunió suficientes firmas para presentar una nueva moción de vacancia. Esta fue debatida y votada el 9 de noviembre. Sorpresivamente, recibió el apoyo mayoritario con 105 votos a favor, 19 en contra, y 4 abstenciones. Hasta la mañana de ese día las declaraciones de algunos líderes partidarios hacían pensar que no habría votos suficientes para la vacancia. Por la tarde se obtuvo ese resultado apabullante.

La destitución del presidente sorprendió a la población, más aún frente a la decisión de Vizcarra de aceptarla inmediatamente. Esa misma noche empezaron los cacerolazos contra el Congreso, lo que dio inicio a una de las olas de protesta ciudadana más masivas y generalizadas de las últimas décadas. El 10 de noviembre, el presidente de la Mesa Directiva del Congreso, Manuel Merino de Lama, juramentó como nuevo presidente de la República, tal como lo prevé la Constitución. ${ }^{8}$ Merino, miembro del partido Acción Popular y elegido previamente en otras dos oportunidades como congresista por el departamento de Tumbes, era un político poco conocido a nivel nacional. Rápidamente se perfiló como uno de los principales operadores de la vacancia presidencial.

La conformación del gabinete de Merino no contribuyó a disminuir la protesta ciudadana. "Ultraconservadores, exfuncionarios del APRA y responsables de gremios empresariales integran gobierno de Merino", titulaba un importante medio de comunicación digital para calificar a su gobierno (Ojo Público 2020). Las protestas ciudadanas se dieron en Lima y las principales ciudades del país, casi sin cesar durante una semana. Decenas de miles de peruanos, sobre todo jóvenes, salieron en contra de lo que podía ser descrito como un "golpe legislativo" por el abuso de la figura de vacancia presidencial como se ha dado antes en otros países de América Latina (Pérez-Liñan 2007). En el centro de Lima, la represión policial fue brutal, llevando a la muerte de los jóvenes Inti Sotelo

32 votos a favor, 78 en contra y 15 abstenciones.

Martin Vizcarra era primer vice-presidente de la República al momento de reemplazar a Pedro Pablo Kuczynski a la Presidencia, y Mercedes Araoz, quien ocupaba la segunda vice-presidencia, había renunciado al cargo en octubre 2019 tras la disolución del Congreso. 
y Bryan Pintado y a centenares de heridos. Esta conducta contrastaba con la actuación de la policía en otras protestas urbanas, donde desde hace años se ejercía un mayor control en el uso de la fuerza. La indignación de la población fue casi unánime frente al uso de armas de fuego por los efectivos policiales y las muertes de los jóvenes. El gobierno de Merino fue reconocido tímidamente solo por algunos jefes de estado de la región. Finalmente, renunció el 15 de noviembre, ni siquiera una semana luego de asumir el mando.

Cabe señalar que el Tribunal Constitucional decidió resolver pocos días después de la renuncia de Merino la demanda competencial interpuesta por el Ejecutivo en septiembre al momento del primer intento de vacancia. En su sentencia el Tribunal declaró improcedente la demanda competencial, negándose a dar una interpretación al causal de "incapacidad moral permanente" por considerar que ya no tenía sentido hacerlo al no haber procedido ese intento de vacancia. La decisión fue duramente criticada pues no tocaba el fondo de la cuestión. Este causal ha sido una espina en el zapato del orden constitucional, volviendo recurrentemente a ser invocada por actores cuyos motivos han sido más de una vez poco escrupulosos. Según varios juristas, al momento de entrar en la Constitución a mediados del siglo XX, este causal de vacancia significaba básicamente incapacidad mental y si bien se ha ampliado su uso debe estar sujeto a criterios excepcionales y que sean armoniosos con el artículo que restringe la responsabilidad penal del presidente (García Chavarri 2020). Reaccionando a la sentencia del Tribunal Constitucional, el Ministerio de Justicia se pronunció en su cuenta de Twitter: “Desde el Poder Ejecutivo consideramos que el supremo intérprete de la Constitución ha perdido una gran oportunidad para clarificar los alcances de la causal de vacancia presidencial por "permanente incapacidad moral"'". ${ }^{9}$ Este punto es también importante para entender la incertidumbre que rodea las reglas que regulan la relación entre el Ejecutivo y el Legislativo, tal como abordaremos en la conclusión. Con reglas tan abiertas es muy difícil que sean interpretadas de manera consensuada y con fronteras legítimas para su uso.

Al renunciar Merino, también renunció la mesa directiva del Congreso. La línea de sucesión presidencial presentó un nuevo impase. El mismo día de la renuncia, el Congreso tuvo una sesión extraordinaria para elegir una nueva Mesa directiva. Las negociaciones duraron varias horas, y finalmente se aprobó una lista multipartidaria liderada por el ingeniero Francisco Sagasti Hochhausler. Tal como esta previsto en la Constitución, Sagasti juramentó como cuarto presidente de la República en tan solo 4 años. Quien era presentado como candidato a la vicepresidencia en las elecciones del 2021 en la lista del Partido Morado, aceptó el reto de ser presidente transitorio por un periodo de ocho meses y renunció a su candidatura para preservar la neutralidad del gobierno. 
Así, el año 2020 terminó en una situación política aún más preocupante que la del primer mes, con una crisis económica sin precedente en las últimas décadas, un gobierno de profesionales destacados pero con poca experiencia política, y un manejo de la emergencia sanitaria que no llegó a convencer luego de las primeras semanas. La caída de Vizcarra y el cambio de gabinete que generó interrumpieron los esfuerzos desplegados para la negociación de los contratos con las farmacéuticas productoras de vacunas contra la COVID-19. Por ejemplo, al final del 2020, las negociaciones con la empresa Pfizer todavía no concluían. Recién se firmaría el contrato en febrero del 2021 (Gestión 2021). El acuerdo con la farmacéutica china Sinopharm, vinculado a la participación del Perú en uno de los ensayos clínicos de su vacuna, dio lugar a cierta esperanza de acceder a la vacuna. Pero los avances alcanzados resultaron amenazados cuando se reveló que el entonces presidente Vizcarra, algunos de sus ministros y otros funcionarios públicos, además de los investigadores y rectores de las universidades responsables del ensayo clínico se vacunaron de manera irregular y secreta durante el desarrollo del ensayo clínico de Sinopharm en 2020 y principios del 2021. El "Vacunagate" que estalló en febrero 2021, dejó un sabor muy amargo para la mayoría de la población que, en su momento, confiaba en que el gobierno de Martin Vizcarra trabajaba arduamente para vencer la pandemia.

Más allá del atraso en la gestión de la vacuna contra la COVID-19, el nuevo equipo de gobierno del presidente Sagasti experimentó otro gran reto socio-político en las primeras semanas de instalarse. Un paro de trabajadores del sector agroexportador se armó para demandar la revisión de una serie de incentivos para la promoción de esta actividad aprobados en el año 2000 (Ley N. ${ }^{\circ} 27360$ ). Estos fueron renovados en varias oportunidades, la última vez a pedido del gobierno de Martín Vizcarra. El paro incluyó bloqueo de carreteras en diversos valles costeros, especialmente en los departamentos de Ica y La Libertad. Finalmente el Congreso, en diálogo con el Poder Ejecutivo, realizó modificaciones sustantivas al régimen, mostrando que incluso instituciones económicas que gozaban de prestigio se han puesto en discusión en la situación actual.

\section{CAMBIOS EN LA RELACIÓN EJECUTIVO LEGISLATIVO, ¿CAMBIOS EN LA CONTINUIDAD POLÍTICA?}

Como discutimos en la introducción diversos autores han llamado la atención sobre ciertas continuidades en la política peruana que pueden parecer sorprendentes dada la debilidad de los partidos políticos y otros actores organizados. En realidad, varios de estos elementos, como el control del Poder Ejecutivo sobre el Congreso, las protestas desarticuladas, o la resistencia del modelo económico adoptado en los noventa, se explican en parte por esta debilidad de los actores políticos. El mayor peso relativo de empresarios, tecnócratas y en general de actores estatales del Ejecutivo que apoyan el statu quo, contribuyó a mantener un sistema político inaugurado desde los años noventa. A pesar 
de tener elecciones en las que opciones de cambio moderado o más profundo obtuvieron buenos resultados, a pesar de que existe conflictividad a nivel local en especial vinculada a temas socioambientales, la continuidad ha estado más presente que el cambio. Esta situación es muy problemática en términos democráticos, por supuesto, dada la débil capacidad del sistema político de representar intereses colectivos y por el rol predominante de actores privados e ilícitos con gran influencia en detrimento de otras preferencias.

Un cambio en esta continuidad ha sido la pérdida de poder del Ejecutivo sobre el Legislativo y, más precisamente, sobre la política en general. Paradójicamente esta continuidad se vio afectada cuando dos partidos vinculados a la defensa y continuidad del modelo económico pasan a la segunda vuelta electoral en 2016. El triunfo en la elección presidencial de una derecha con fuertes vínculos empresariales, como la de Kuczynski, y la hegemonía en el Congreso de una derecha popular que reconoce al modelo económico como parte de su legado, como la de Fujimori, no dio lugar al fortalecimiento del statu quo como algunos esperaban. Por el contrario, el conflicto entre estas dos derechas que se bifurcan llevó a que se produjera una serie de quiebres que terminaron afectando la posición del Ejecutivo frente al Congreso (Meléndez 2019). Para entender ese quiebre en el poder del Ejecutivo hay que ponderar este enfrentamiento sumado a la composición del Congreso, con un gobierno en minoría y un partido de oposición mayoritario.

Comencemos por el conflicto, que marcó el tono desde la instalación del Congreso y juramentación del presidente. Un Fujimorismo muy radicalizado tanto por su derrota como por su necesidad de detener las investigaciones que lo involucraban con el caso Lava Jato, atacó al Ejecutivo y sus políticas. Cada vez se hizo más difícil conseguir ministros de prestigio dispuestos a sufrir ese maltrato. En términos de control político abundaron las citaciones a funcionarios del Ejecutivo. No es correcto evaluar esta crispación solo midiendo los ministros citados o las interpelaciones realizadas. Estas interacciones venían cargadas de desplantes y amenazas al gobierno. Si bien el Fujimorismo fue más cuidadoso en lo que respecta a la política económica, también tuvo decisiones duras que afectaron al Ministerio de Economía (MEF), incluso censurar a un ministro y debilitando la posición del MEF frente a la Comisión de Presupuesto y sus pedidos de gasto público. Así, cambió la relación entre el Ejecutivo y el Congreso utilizando una serie de recursos de control político y modificando el control sobre temas de presupuesto, y si bien en teoría hay una dimensión democrática positiva en este mayor control por parte de los parlamentarios, las formas y los objetivos de la mayoría distaron de ser democráticos.

Pero no solo fue el enfrentamiento entre el Fujimorismo y el gobierno. El resultado de la elección, con un primer lugar bastante arriba y un segundo lugar con pocos votos, es lo que hizo posible que esta enemistad tuviera consecuencias tan profundas. Desde el año 2001 los partidos de gobierno han tenido bancadas que les permitían, con negociaciones y alianzas, reducir los riesgos de interpelaciones, censuras y vacancia presidencial. Toledo (45 / 120 escaños), García (36 / 120 
escaños), Humala (47 / 130 escaños) lograron mantener un mayor control del Congreso. Kuczynski (18 / 130 escaños) no solo tenía una bancada más pequeña, sino que la mayoría la tenía el perdedor de la elección; ni siquiera formando alianzas, como García o Toledo, podía controlar las dinámicas del Congreso. Esa mayoría controló las comisiones más importantes y la agenda del pleno del Congreso. La radicalidad de la oposición mayoritaria, y la debilidad numérica del Ejecutivo para contenerla debilitaron la institucionalidad del Poder Ejecutivo.

Es así que en el 2020 esta nueva dinámica ya parecía haberse instalado en el país, el libro de jugadas del Congreso fue apropiado por sus nuevos integrantes. Las reglas de relación entre poderes de la Constitución que ha regido al país desde 1993 fueron reinterpretadas o usadas con más frecuencia (o en forma novedosa) desde el 2016. La elección del nuevo Congreso fragmentado en bancadas pequeñas no atemperó la relación con el Ejecutivo. Al revés, la llegada de representantes inexpertos, algunos más radicales, y otros vinculados a la agenda de intereses económicos particulares, dio lugar a una mezcla explosiva en el marco de la pandemia. Y este problema se acentúa pues esta vez el Ejecutivo ni siquiera presentó una lista para la elección Congresal, asumiendo que la popularidad del presidente Vizcarra y la relación cordial con algunas bancadas, bastaría para contener al nuevo Congreso (ver gráficos 5 y 6). En realidad, se mantuvo y agravó el uso de mecanismos de control horizontal contra el Ejecutivo, pero, como se ha discutido antes, se sumó la aprobación de una serie de leyes buscando responder a las grandes necesidades de la pandemia y a mayores demandas presupuestales. El Ejecutivo carecía de recursos institucionales (cuestión de confianza) para detenerlos pues la Constitución prohíbe la disolución del Congreso en el último año de gobierno. Pero además no tenía bancada para defender sus intereses y detener estas normas.

Gráfico 5. Nivel de Aprobación Vizcarra (2019-2020)

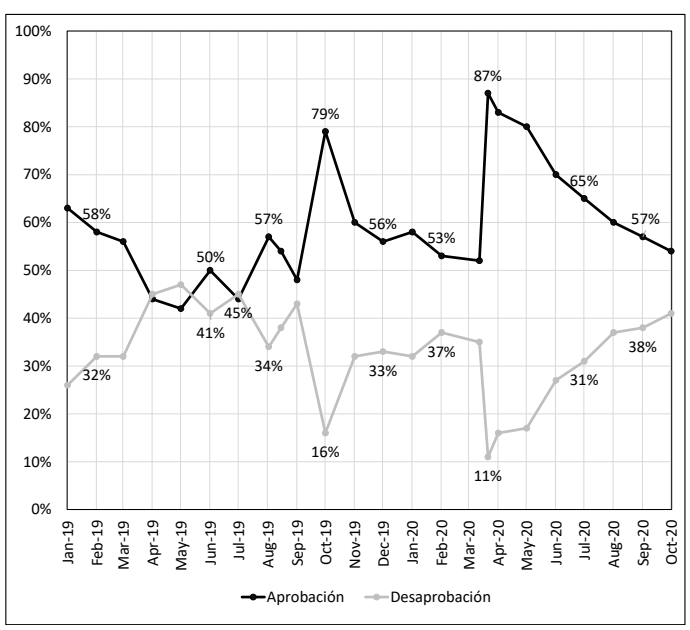

Fuente: Elaboración propia en base a datos de IPSOS. 
Gráfico 6. Nivel de Aprobación Congreso (2019-2020)

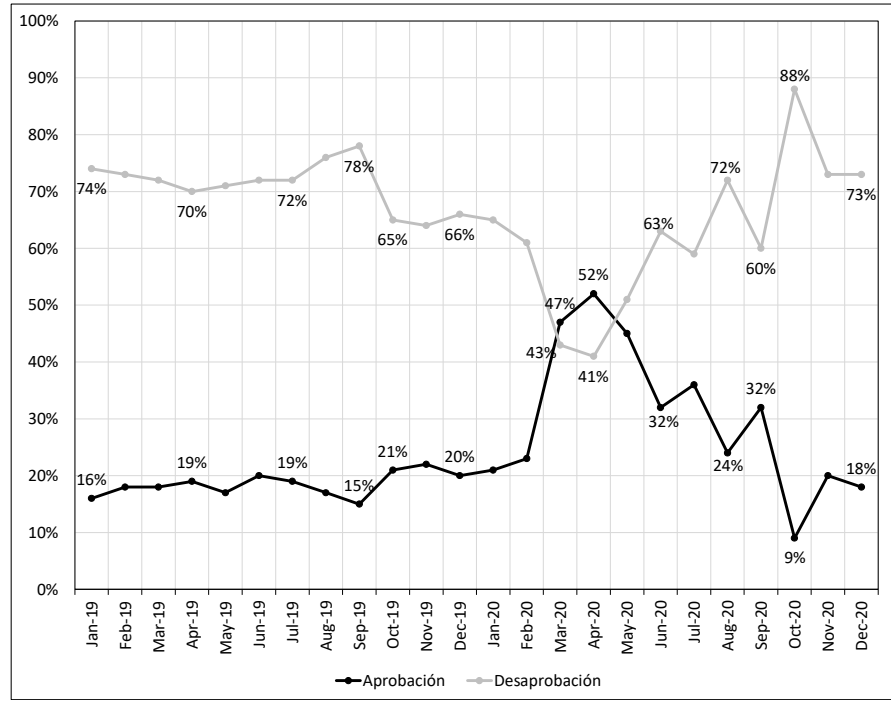

Fuente: Elaboración propia en base a datos de IPSOS.

El problema con este súbito interés en los necesitados es que se aprobaron una serie de medidas poco técnicas y que afectaban la caja fiscal. El MEF ya no pudo detener estas normas, sino que el Ejecutivo tuvo que acudir al Tribunal Constitucional a cuestionar estas leyes. Estas normas cuestionan tanto el arreglo institucional de un MEF cuidando el déficit y la economía como algunos supuestos del modelo económico, como los intentos de control de precios o las pensiones. Se observa, entonces, un Congreso más interesado en la economía y un Ejecutivo con menos poder para controlarlo. En paralelo, el perfil de los ministros seguía disminuyendo. Cabe señalar que Martín Vizcarra fue también responsable de esta mediocrización del Ejecutivo al no utilizar su popularidad para formar gabinetes de mayor peso. Es posible que su necesidad de contar con ministros de confianza en áreas clave por temor a las investigaciones en su contra contribuyera a este desinterés por reforzar al Ejecutivo.

Con respecto a la continuidad, hay también un cambio observado en el periodo, aunque todavía sea prematuro concluir que se trata de algo más profundo: las protestas observadas en noviembre y diciembre. Primero, la movilización ciudadana de noviembre contra la decisión de vacar al presidente Vizcarra y luego pidiendo la renuncia de Manuel Merino fue descentralizada y amplia. Es un cambio, en su magnitud y coordinación, con lo que ha sido la forma de protestar en el Perú incluso en temas altamente sensibles como la concesión del indulto a Alberto Fujimori por parte del gobierno de Kuczynski en el 2017. Y segundo, la protesta vinculada a la agroexportación, que también motivó una protesta articulada en varios valles de la costa. 
Es muy pronto para saber si entramos en una dinámica de protesta distinta en el país. Las razones que han dado lugar a protestas desarticuladas y localizadas todavía subsisten. Sin embargo, ambos eventos sí tienen relevancia por distintas razones. En el primer caso, muestra que la ciudadanía tiene límites en su tolerancia al comportamiento errático y abusivo de los actores políticos; demuestra que las protestas masivas sí eran posibles en el Perú. En el segundo, nos muestra que aspectos centrales de la política económica pueden ser cuestionados desde la calle y que tendrán receptividad en un Congreso que se apresura a alinearse con esas demandas.

Por todo ello, creemos que el año 2020 profundiza un proceso que nos coloca en una trayectoria distinta a la del 2016. El Ejecutivo tiene menos peso político que hace cinco años, el Congreso lo ha ganado y usa su poder para tomar medidas contra el manejo económico que antes estaba controlado por el MEF. No minimizamos los rasgos que mantiene el sistema político peruano, ni negamos la posibilidad de que podamos volver hacia un equilibrio similar al de los años previos si por ejemplo el ganador de la elección del 2021 logra una bancada de buen tamaño que aleje la vacancia y permita un mejor manejo económico. Pero hoy parece más probable que nuestra continuidad sea muy distinta a la de hace cinco años. Congresos fragmentados, con bancadas que se dan la mano en temas presupuestarios, serán una amenaza para Ejecutivos que llegarán al poder con bancadas relativamente pequeñas (y que a su interior también tendrán integrantes que buscarán aprovechar la debilidad del Ejecutivo para controlar el presupuesto). Las armas nucleares de la disolución y la vacancia ya se han instalado como recursos comunes, de la disputa política. Muy pronto para saber si estas dinámicas llevarán a otros cambios en otras dimensiones, pero el peso del Ejecutivo ha sido clave para una serie de dinámicas del sistema político peruano en lo que respecta a la estabilidad y el manejo económico. Hoy ese peso ha cambiado, sin que haya sido modificado las reglas constitucionales. Parafraseando a Legión Urbana, el futuro ya no es lo que era antiguamente. $\mathrm{Y}$ se ve peor.

\section{CONCLUSIÓN: UN QUINQUENIO CON MALOS AUGURIOS}

Al momento de terminar la redacción de este artículo, la elección del 11 de abril apunta a un resultado donde los candidatos que pasen a segunda vuelta no obtendrán ni remotamente los porcentajes de elecciones anteriores. A un mes de la elección, hay apenas nueve puntos (13\%) entre el primer candidato en las encuestas y el décimo (4\%). Por ello, muy probablemente tendremos de nuevo un Congreso fragmentado en más de siete bancadas y un gobierno minoritario en el Congreso que mantendrá abierto el riesgo de una vacancia presidencial. Todo hace pensar que seguirán las tensiones vividas en los últimos años. El gobierno, por otro lado, también contará con su arma nuclear respectiva: la amenaza de la disolución si se traba su desempeño. Veremos, además, a congresistas con menos experiencia política que el Congreso anterior, pues 
ya opera la prohibición de reelección congresal. Y es probable que aumente la polarización pues es casi seguro que contaremos con una bancada de derecha radical relevante.

Optimistamente algunos líderes de opinión pensaron que el 2020 serviría para reducir en lo posible la forma amplia en que se interpretan los mecanismos de vacancia, la cuestión de confianza y la eventual disolución del Congreso, en el mejor de los casos legislando al respecto. Como se mencionó antes, el Tribunal Constitucional perdió una oportunidad de pronunciarse sobre los requisitos y límites de la vacancia presidencial y la sentencia sobre disolución del Congreso sigue siendo bastante amplia como para establecer límites claros. Lo que queda como lección es que un acto grave, definido como tal por el propio Congreso, puede ser suficiente para vacar a un presidente si se tienen los votos necesarios.

Para colmo de males, en un Congreso fragmentado y con muchos partidos sin agendas programáticas, es probable que se reparta la elección de los seis magistrados constitucionales que toca renovar y se elija a jueces más abiertos a las preferencias del legislativo. Ello limitará el control que hoy por hoy le permite al Ejecutivo actuar por medio de demandas constitucionales en contra de los embates populistas del Congreso.

Toda esta situación apunta a que tendremos un periodo presidencial tenso y conflictivo, donde será muy difícil para el Ejecutivo conseguir ministros de calidad que puedan empujar reformas y mejorar el desempeño del gobierno así como dar seguridad y estabilidad a la burocracia en el desarrollo de políticas. Es posible que tengamos cinco años más de pugnas, en el peor de los casos debilitando al Ejecutivo todavía más e incrementando el poder del Congreso para imponer pedidos y agendas particularistas. $\mathrm{O}$ también son posibles nuevas tensiones que pueden llevarnos hacia una radicalización del Ejecutivo buscando la disolución de un Congreso que probablemente también sea impopular. Ello podría abrir la puerta a una mayor concentración de poder, pues difícilmente un presidente volverá a abstenerse de postular una lista parlamentaria a la nueva elección congresal como lo hizo Vizcarra en 2020. Todo hace pensar que estamos viviendo procesos que marcan un cambio en la continuidad peruana post-noventa, donde la debilidad del próximo Ejecutivo pueda tener un mayor impacto negativo sobre la gobernabilidad que en las décadas previas.

Lo que se va debilitando no constituían grandes virtudes del sistema político peruano, criticado con justicia por sus limitaciones democráticas. La debilidad partidaria ha hecho difícil construir una mejor representación, no se ha avanzado en políticas de bienestar social como en otros estados de la región y no observamos procesos sostenidos de reforma del Estado para hacerlo más eficiente y democrático. Sin embargo, había ciertas condiciones que permitían estabilidad económica, un control del déficit público y límites a políticas populistas. Mucho de eso ha cambiado, abriendo la puerta a tensiones de diverso tipo dependiendo de quién gane la presidencia en 2021. Peor si se suma a ello la 
polarización ideológica. Esto no implica lamentar la pérdida de lo que se tenía, pero sí preocuparse para evitar que estos cambios nos conduzcan a algo peor.

\section{REFERENCIAS}

Andina. 2021, 4 de enero. "PUCP facilitará 275 ventiladores mecánicos al Minsa para atención del covid-19." Agencia Andina. Recuperado el 9 de julio de 2021 de https:/ / andina. pe/agencia/noticia-pucp-facilitara-275-ventiladores-mecanicos-al-minsa-para-atencion-del-covid19-828607.aspx

Awapara, Omar. 2018. "The Geography of Free Trade: Explaining Variation in Trade Policy in Latin America." Ph.D. Dissertation, University of Texas at Austin, August 2018. Retrieved from https://repositories.lib.utexas.edu/bitstream/handle/2152/68472/ AWAPARA-DISSERTATION-2018.pdf?sequence=1\&isAllowed $=y$

Berganza, Isabel. 2020, 16 de julio. "Nuestros Migrantes Internos y el Covid." Diario El Peruano. Recuperado el 9 de julio de 2021 de https://elperuano.pe/noticia/99695-nuestros-migrantes-internos-y-el-covid-19

CEPAL. 2020. "Salud y economía: una convergencia necesaria para enfrentar el COVID-19 y retomar la senda hacia el desarrollo sostenible en América Latina y el Caribe." Informe Covid 19 CEPAL-OPS. Recuperado el 9 de julio de https:/ / repositorio.cepal.org/ bitstream/handle/11362/45840/4/S2000462_es.pdf

CEPAL. 2021. "Balance Preliminar de las Economías de América Latina y del Caribe 2020." Documento anual de la División de Desarrollo Económico de la Comisión Económica para América Latina y el Caribe (CEPAL), Santiago. Recuperado el 9 de julio de 2021: https://repositorio.cepal.org/bitstream/handle/11362/46501/112/S2000990_es.pdf

CIUP. 2020. “¿Cuál debe ser el propósito del sistema previsional durante la crisis económica y sanitaria?" Centro de Investigación de la Universidad del Pacífico Lima. Recuperado el 9 de julio de 2021: https:/ / ciup.up.edu.pe/analisis/cual-debe-ser-proposito-sistema-previsional-durante-crisis-economica-sanitaria /

Colegio Médico del Perú. 2021. "Médicos con Covid-19 Positivo. Autorreporte del Colegio Médico del Perú". Recuperado el 9 de Julio de 2021 de https://www.cmp.org.pe/ medicos-con-covid-19-positivo-autoreporte/

Dargent, Eduardo y Paula Muñoz. 2016. "Peru: A Close Win for Continuity." Journal of Democracy $27(4): 145-58$.

Dargent, Eduardo. 2015. Technocracy and Democracy in Latin America. The Experts Running Government. Cambridge: Cambridge University Press.

Defensoría del Pueblo. 2020. "Crisis de oxígeno para pacientes de COVID-19: Alternativas de solución." Serie Informes Especiales $N^{0}$ 017-2020-DP, Defensoría del Pueblo, Lima. Recuperado el 9 de julio de 2021: https://www.defensoria.gob.pe/wp-content/ uploads/2020/06/Serie-Informes-Especiales-N\%C2\%BA-017-2020-DP.pdf

Durand, Francisco. 2006. El Problema Del Fortalecimiento Institucional. En Construir Instituciones: Democracia, Desarrollo Y Desigualdad en el Perú desde 1980, editado por John Crabtree. Lima: CIUP, PUCP e IEP, 189-210.

García Chavarri, Abraham. 2020. “Tres maneras de conceptualizar la figura de la permanente incapacidad moral del presidente de la República como causal de vacancia en el cargo." Boletín IDEHPUCP 17-11-2020. Recuperado el 9 de julio de 2021 de https:/ / idehpucp.pucp.edu.pe/notas-informativas/tres-maneras-de-conceptualizar-la-figura-de-la-permanente-incapacidad-moral-del-presidente-de-la-republica-como-causal-de-vacancia-en-el-cargo/

Gestión. 2020, 31 de diciembre. "Perú cierra el 2020 con 94,000 muertes más que las registradas en años previos." Recuperado el 9 de julio de 2021 de https:/ /gestion.pe/peru / peru-cierra-el-2020-con-94000-muertes-mas-que-las-registradas-en-anos-previos-noticia/ 
Gestión. 2021, 4 de febrero. "Perú firma acuerdo por 20 millones de vacunas con Pfizer y primeras entregas serán en marzo y abril." Recuperado el 9 de julio de 2021 de https://gestion.pe/peru/vacunas-covid-19-peru-firma-acuerdo-por-20-millones-devacunas-con-pfizer-y-primera-entregas-seran-en-marzo-y-abril-nndc-noticia /

Herrera-Añazco, Percy, et al. 2021. "Some Lessons That Peru Did Not Learn Before the Second Wave Of COVID-19." International Journal of Health Planning and Management 1: 995-998.

INEI. 2021. "Situación del Mercado Laboral en Lima Metropolitana." Informe técnico del Instituto Nacional de Estadística e Informática. Recuperado el 9 de julio de https:/ /www. inei.gob.pe/media/MenuRecursivo/boletines/02-informe-tecnico-mercado-laboral-nov-dic2020-ene2021.pdf

Lavado, Pablo y César Liendo. 2020. 29 de mayo. “COVID-19, Pobreza Monetaria y Desigualdad." Foco Económico. Recuperado el 9 de julio de 2021 de https://focoeconomico. org/2020/05/29/covid-19-pobreza-monetaria-y-desigualdad/

Levitsky, Steven y Max Cameron. 2003. "Democracy Without Parties? Political Parties and Regime Change in Fujimori's Peru." Latin American Politics and Society 45: 1-33.

Meléndez, Carlos y Carlos León. 2010. "Perú 2008: El Juego de Ajedrez de la Gobernabilidad en Partidas Simultáneas." Revista de Ciencia Política 29(2): 591-609.

Meléndez, Carlos. 2019. “La Derecha que se Bifurca. Las Vertientes Populista-Conservadora y Tecnocrática-Liberal en Perú Post-2000." Colombia Internacional 99: 3-27.

Ojo Público. 2020, 12 de noviembre. "Ultraconservadores, Ex Funcionarios del APRA y Representantes de Gremios Empresariales Integran Gabinete de Merino." Recuperado el 9 de julio de 2021 de https://ojo-publico.com/2235/manuel-merino-juramenta-un-polemico-gabinete-en-el-peru

Oyola, Alfredo Enrique. 2021. "Desigualdad en la Distribución de Médicos en el Perú." Revista Cubana de Salud Pública 47(1): 1-16.

Paredes, Maritza y Daniel Encinas. 2020. "Perú 2019: Crisis Política y Salida Institucional." Revista de Ciencia Política 40(2): 483-510.

Pérez-Liñan, Anibal. 2007. Presidential impeachment and the new political instability in Latin America. Cambridge: Cambridge University Press.

PNUD. 2020. "Reporte de la Sexta Reunión (5-6-2020)." Grupo de Expertos y Expertas: La Economía de la Pandemia y Protección Social en América Latina. Recuperado el 9 de julio de 2021 de https://www.latinamerica.undp.org/content/rblac/es/home/coronavirus/social-protection-to-face-the-pandemic/relatoria-6.html

Podestá, Andrea. 2020. "Gasto público para impulsar el desarrollo económico e inclusivo y lograr los Objetivos de Desarrollo Sostenible." Serie Macroeconomía del Desarrollo $N^{\circ} 214$ de la Comisión Económica para América Latina y el Caribe (CEPAL). Recuperado el 9 de julio de 2021 de https://www.cepal.org/sites/default/files/publication/files/46276/S2000670_es.pdf

Ponce de León, Zoila y Luis García. 2019. “Perú 2018: La Precariedad Política en Tiempos de Lava Jato." Revista de Ciencia Política 39(2): 341-365.

RPP. 2021, 1 de enero. "Hay 7600 Profesionales de Enfermería Contagiados con Covid-19." Recuperado el 9 de Julio de 2021 de https://rpp.pe/peru/actualidad/coronavirus-en-peru-liliana-la-rosa-hay-7-600-profesionales-de-enfermeria-contagiados-covid-19-noticia-1318360

Salud con Lupa. 2017. "Centro de Datos Coronavirus". Recuperado el 9 de Julio de 2021 de https://saludconlupa.com/series/coronavirus/centro-de-datos/capacidad-de-unidades-de-cuidados-intensivos /

Tanaka, Martín y Sofía Vera. 2008. "El "neodualismo" de la política peruana." Revista de Ciencia Política 28(1): 347-365.

UNMSM. 2021, 26 de enero. "Sanmarquinos Desarrollan Primera Planta de Producción Nacional de Pruebas Moleculares." Universidad Nacional Mayor de San Marcos. Recuperado el 9 de Julio de 2021 de https://www.unmsm.edu.pe/noticias-y-eventos/ 
noticias/noticia-detalle/covid-19-sanmarquinos-desarrollan-primera-planta-de-produccion-nacional-de-pruebas-moleculares

Vergara, Alberto y Daniel Encinas. 2016. “Continuity by Surprise. Explaining Institutional Stability in Contemporary Peru." Latin American Research Review 51(1): 159-180.

Zavaleta, Mauricio. 2014. Coaliciones de Independientes. Las Reglas No Escritas de la Política Electoral. Lima: IEP.

Recibido: 30 de marzo de 2021

Aceptado: 15 de julio de 2021

Eduardo Dargent. Profesor Principal del Departamento de Ciencias Sociales de la Pontificia Universidad Católica del Perú. Doctor (PhD) en ciencia política de la Universidad de Texas en Austin y Maestría en Filosofía Política de la Universidad de York (Reino Unido). Sus temas de investigación son la política de las políticas públicas, economía política, el Estado en América Latina y partidos políticos. Su libro Technocracy and Democracy: The Experts Running Government (Nueva York: CUP) fue publicado el 2016. Ha publicado artículos en las revistas Comparative Politics, Journal of Latin American Politics, Revista de Ciencia Política, entre otras. Correo electrónico: edargent@pucp.edu.pe

Stéphanie Rousseau. Profesora Asociada del Departamento de Ciencias Sociales de la Pontificia Universidad Católica del Perú. Doctora (PhD) en ciencia política de la McGill University (Montreal) y Maestría en estudios internacionales de la Carleton University (Ottawa). Recibió en 2016 el Premio José-María Arguedas del mejor artículo sobre Perú de la Latin American Studies Association-Sección Perú. Trabaja sobre el género, la etnicidad, la ciudadanía, los movimientos sociales, y la política de las políticas públicas en Perú y Bolivia. Su libro más reciente, en coautoría, es Indigenous Women's Movements in Latin America: Gender and Ethnicity in Peru, Mexico and Bolivia (Palgrave 2017). Ha publicado artículos en las revistas Latin American Research Review, Journal of Latin American Politics, Latin American Politics and Society, entre otras. Correro electrónico: srousseau@pucp.pe 